\title{
Experimental chronic jet lag promotes growth and lung metastasis of Lewis lung carcinoma in C57BL/6 mice
}

\author{
MINGWEI WU ${ }^{1,2,6}$, JING ZENG ${ }^{1,3}$, YANFENG CHEN ${ }^{1,4}$ ZHAOLEI ZENG ${ }^{1,2}$, JINXIN ZHANG $^{1,5}$,

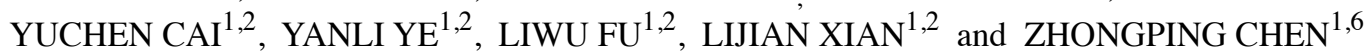 \\ ${ }^{1}$ State Key Laboratory of Oncology in South China; Departments of ${ }^{2}$ Research, ${ }^{3}$ Pathology, and ${ }^{4}$ Head and Neck Cancer, \\ Cancer Center, Sun Yat-Sen University; ${ }^{5}$ Department of Medical Statistics and Epidemiology, Sun Yat-Sen University; \\ ${ }^{6}$ Department of Neurosurgery, Cancer Center, Sun Yat-Sen University, Guangzhou, Guangdong, P.R. China
}

Received December 8, 2011; Accepted January 17, 2012

DOI: $10.3892 /$ or.2012.1688

\begin{abstract}
Circadian rhythm has been linked to cancer genesis and development, but the detailed mechanism by which circadian disruption accelerates tumor growth remains unclear. The purpose of this study was to investigate the effect of circadian disruption on tumor growth and metastasis in male C57BL/6 mice, using an experimental chronic jet lag model. Lewis lung carcinoma cells were inoculated into both flanks of the mice following 10 days of exposure to experimental chronic jet lag or control conditions. The effects on tumor growth and lung metastasis were assessed, and the effect on gene expression was detected using cDNA microarrays and real-time quantitative RT-PCR. Tumors grew faster in the experimental chronic jet lag mice compared to the control mice $(\mathrm{P}=0.004)$. Lung metastases were found in 10 out of 24 mice in the chronic jet lag group, but only in 3 out of 24 mice in the LD group $(\mathrm{P}=0.023)$. Microarray data showed that in both liver and tumors circadian disruption altered the expression of genes, including those related to the cell cycle, apoptosis, the immune response and metastasis suppressor genes. The expression of the NDRG1 gene was suppressed by chronic jet lag. We conclude that circadian disruption can promote tumor progression and metastasis by affecting the expression of both tumor-related genes and metastasis suppressor genes.
\end{abstract}

\section{Introduction}

Most living organisms, from cyanobacteria to plants, insects, and mammals, are capable of displaying spontaneously sustained oscillations with a period close to $24 \mathrm{~h}$, known as 'circadian rhythm'. Studies have found that circadian rhythms

Correspondence to: Dr Mingwei Wu or Zhongping Chen, State Key Laboratory of Oncology in South China, Cancer Center, Sun Yat-Sen University, Guangzhou, Guangdong 510060, P.R. China E-mail:wumw@mail.sysu.edu.cn

E-mail: chenzhp@sysucc.org.cn

Key words: jet lag, circadian rhythm, tumor, metastasis, mouse are governed by a biological clock. The mammalian circadian clock contains three components: input pathways, a central pacemaker and output pathways. The mammalian central pacemaker is located in the suprachiasmatic nuclei (SCN) of the anterior hypothalamus and controls the activity of the peripheral clocks through the neuroendocrine and autonomic nervous systems $(1,2)$. Circadian rhythms govern the rhythmic changes in the behavior and/or physiology of mammals, such as body temperature, blood pressure, hormone production, digestive secretion, neurotransmitter secretion, and even gene expression (1,3-6).

Circadian rhythms have been found to play a very important role in cancer genesis and development. Fu et al showed that mutant mice deficient in the period homolog 2 (Per2) gene, a core circadian-clock gene, had a more marked increase in tumor development after gamma radiation than the wild-type mice $(7,8)$. Wood et al showed that mutation of the Per2 gene accelerated ApcMin ${ }^{+}$tumorigenesis in mice (11). Malignant growth was accelerated by the disruption of circadian coordination that resulted from SCN destruction or experimental chronic jet lag (8-11). In contrast, overexpression of Perl, another core circadian gene, in human cancer cell lines can lead to significant growth reduction (12). Although circadian rhythms have been linked to cancer, the detailed mechanism by which circadian disruption accelerates tumor growth remains unclear.

Therefore, we hypothesized that circadian disruption might disrupt the expression of the clock genes and other tumor-related genes that could lead to a decrease in the level of the antitumor response. This study on Lewis lung carcinoma (LLC) cells investigated the effects of experimental chronic jet lag on growth, lung metastasis, and gene expression, which was detected using the global genomic gene scan.

\section{Materials and methods}

Ethics statement. All research involving animal experiments was approved by the Animal Ethics Committee of the Cancer Center, Sun Yat-sen University (approved ID, NSFC30500589).

Animals and synchronization. A total of 48 male, 4-week-old C57BL/6 mice (Vital River Laboratory Animal Technology 
Co., Ltd., Beijing, China) were housed and kept in an autonomous chronobiological facility (Suzhou Anke Purification Equipment Factory, China) equipped with temperature control $\left(23 \pm 1^{\circ} \mathrm{C}\right)$. Mice were kept in six compartments, each provided with filtrated air $\left(700 \mathrm{~m}^{3} / \mathrm{h}\right)$, but with the potential to apply separate lighting regimens (300 Lux). Four mice were housed per cage. The mice were synchronized to a daily light: darkness ratio of $12 \mathrm{~h}$ light and $12 \mathrm{~h}$ darkness (LD 12:12) for 2 weeks. Food (normal chow, Medical Laboratory Animal Centre of Guangdong, China) and water were supplied ad libitum. The mice were subsequently randomized into two groups: half $(n=24)$ became the experimental chronic jet lag (CJL) group, who were exposed to the jet lag light scheme created by advancing the light onset by $8 \mathrm{~h}$ every $48 \mathrm{~h}$; the remainder $(\mathrm{n}=24)$ served as the unshifted control group (LD), who stayed in the LD 12:12 conditions (Fig. 1) (6).

Body temperature. Rectal temperature was measured twice during the experiment (after 2 weeks of synchronization and after 10 days of jet lag) using a digital thermometer (Omron $^{\mathrm{TM}}$-MC612, Omron Dalian Co., Ltd., China). Rectal temperatures of all of the mice in the LD group were measured at six zeitgeber time (ZT) points (ZT3, 7, 11, 15, 19, and 23) within $48 \mathrm{~h}$ (where ZT12 was lights off). In the CJL group, the rectal temperatures of the mice were measured at the same local time (Beijing time) and corresponding time points were labeled as ZT3x, 7x, 11x, 15x, 19x and 23x (Fig. 1).

Tumor inoculation and measurement. The LLC cell line was obtained from the Cancer Centre, Sun Yet-sen University and cultured in Dulbecco's modified Eagle's medium (DMEM) supplemented with $10 \%$ fetal bovine serum (FBS), penicillin $(100 \mathrm{U} / \mathrm{ml})$, and streptomycin $(100 \mathrm{U} / \mathrm{ml})$. Ten days after the onset of the experimental CJL, LLC cells $\left(0.2 \mathrm{ml}, 5 \times 10^{6} / \mathrm{ml}\right)$ were injected subcutaneously into both flanks of mice. Two perpendicular diameters $(\mathrm{mm})$ of each tumor were measured every 4 days with a caliper. Tumor volume $\left(\mathrm{mm}^{3}\right)$ was calculated as: tumor volume $=\left(\right.$ length $\mathrm{x}$ width $\left.{ }^{2}\right) / 2$. The body weights of the mice were monitored during the experiment and no statistically significant difference was found between the LD and CJL groups.

Four mice from the LD group were sacrificed at each time point (ZT3, 7, 11, 15, 19 and 23) and the same number of jet lag mice were sacrificed at the corresponding time points (ZT3x, $7 x, 11 x, 15 x, 19 x$ and 23x; Fig. 1). The lungs, livers and tumors were aseptically removed. The whole lung was fixed with Bouin's fixative. One part of liver and tumor of the host animals were dissected in RNase-free Hank's medium and immediately placed into storage reagent (RNAlater, Ambion, Inc., Austin, TX, USA) on ice, then stored at $-80^{\circ} \mathrm{C}$ until RNA extraction. Another part of the liver and the tumor tissue were placed into $10 \%$ buffered formalin for $24 \mathrm{~h}$. Hematoxylin and eosin (H\&E) stained sections were prepared by standard techniques.

cDNA microarray. The differential display of genes from the liver and tumor samples from two mice in each group at two selected time points (ZT7 and 19, LD group; and ZT7x and 19x, CJL group) was detected using a cDNA gene chip (GeneChip ${ }^{\circledR}$ Mouse Genome 430A 2.0 Array, Affymetrix,
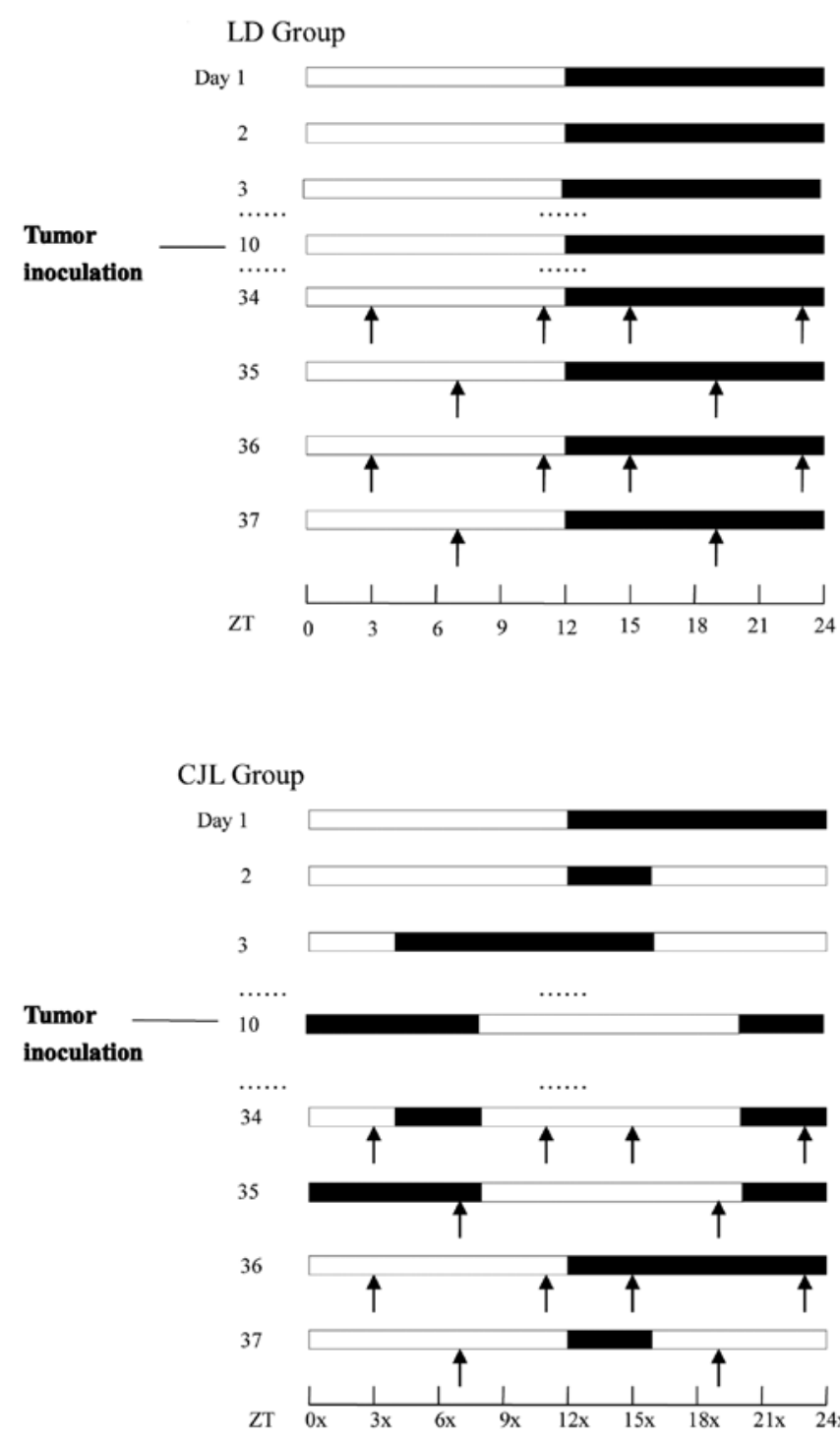

Figure 1. Light regimens: (A) control (LD) group: LD 12:12; (B) chronic jet lag (CJL) group: scheme advancing light onset $8 \mathrm{~h}$ for $48 \mathrm{~h}$. Open and black boxes represent light and darkness respectively. Arrows show the sampling time. Day numbers are the experimental chronic jet lag days. The dash points indicate the light schedule. The inoculation of the tumor was performed on Day 10.

Santa Clara, CA, USA), which is a single array representing $\sim 14,000$ well-characterized mouse genes.

Total RNA was extracted from the frozen liver and tumor specimens using the RNeasy Mini kit ${ }^{\circledR}$ (Qiagen, Valencia, CA, USA) following the instructions of the manufacturer. The amount of RNA was measured spectrophotometrically by the absorbance at $260 \mathrm{~nm}$. Extracted RNA purity was assessed by the ratio of the absorbance at 260 and $280 \mathrm{~nm}\left(\mathrm{OD}_{260 / 280}\right)$. The RNA was stored at $-80^{\circ} \mathrm{C}$ after preparation.

The GeneChip ${ }^{\circledR} 3^{\prime}$ IVT Express kit was used to synthesize first-strand cDNA from total RNA by reverse transcription. This cDNA was then converted into a double-stranded DNA template for transcription. During in vitro transcription, amplified RNA was synthesized and a biotin-conjugated nucleotide was incorporated. The amplified RNA was purified, and then hybridized to the chip. After hybridization, the chip was washed, stained using the GeneChip ${ }^{\circledR}$ Hybridization, Wash, 
and Stain Kit (Affymetrix), and scanned on a chip reader (Affymetrix).

Microarray data analysis. Robust multichip analysis (RMA) was performed using the Affymetrix Expression Console software (Affymetrix). Principal component analysis (PCA) and hierarchical clustering analysis were performed with the Partek GS 6.4 software (Partek ${ }^{\circledR}$ Genomics Suite ${ }^{\mathrm{TM}}$, Partek Inc., St. Louis, MO, USA). The differences in gene expression patterns were designated as significantly different if a $>2$-fold difference was observed between the two groups.

The molecular functions and pathways of the identified genes were analyzed by Ingenuity Pathway Analysis (IPA; Ingenuity 8.5-2803, Ingenuity System Inc., Redwood City, CA, USA) and the Database for Annotation, Visualization and Integrated Discovery (DAVID) v6.7 $(13,14)$.

Real-time quantitative RT-PCR. The selected genes identified using the cDNA microarray were quantified by real-time quantitative RT-PCR. Two-step real-time quantitative RT-PCR was performed. Reverse transcription was performed at $42^{\circ} \mathrm{C}$ for 60 min using the RevertAid First Strand cDNA Sythesis kit (Fermentas, Burlington, ON). Real-time PCR was performed using the Maxima ${ }^{\circledR}$ SYBR-Green qPCR Master Mix (2X) kit (Fermentas). The real-time quantitative PCR conditions were: pre-denaturation at $95^{\circ} \mathrm{C}$ for $10 \mathrm{~min}$, denaturation at $95^{\circ} \mathrm{C}$ for $15 \mathrm{sec}$, annealing at $60^{\circ} \mathrm{C}$ for $35 \mathrm{sec}$, and extension for $30 \mathrm{sec}$ (40 cycles) followed by a reaction as $95^{\circ} \mathrm{C}$ for $15 \mathrm{sec}, 60^{\circ} \mathrm{C}$ for $30 \mathrm{sec}, 95^{\circ} \mathrm{C}$ for $15 \mathrm{sec}$. Primer sequences were as follows: forward 5'-ATGTGCAGCTGATAAAGACTGG-3' and reverse 5'-AGGCCTTGACCTTTTCAGTAAG-3' (m36B4); forward 5'-GTGAAGCAGGTGAAGGCTAATG-3' and reverse 5'-AAGCTTGTAAGGGGTGGTGTAG-3' (mPer2); forward 5'-AAGAGTTGTGAGGCTGGCAC-3' and reverse 5'-GCTCAAACTTCTGGCCTTTG-3' (PTPRC); forward 5'-AGCGGCAGGTTACATTCAAA-3' and reverse 5'-CAA GTTTTGGTGGCACACAG-3' (CD44); forward 5'-GTGAG GATGACAGGACGGTT-3' and reverse 5'-AAAAGGGGAGA GCATCACTG-3' (NDRG1). The specificity of the PCR products was assessed by the melting curve analysis, and by agarose gel electrophoresis, to check for the presence of non-specific products and to confirm that the size of the product corresponded to that of the expected amplicon. The relative levels of each mRNA of the genes of interest were normalized to the corresponding 36B4 RNA levels by the following formula: relative mRNA level $=2^{-\Delta \Delta \mathrm{Ct}}$. The $\mathrm{Ct}$ (cycle threshold) is defined as the number of cycles required for the fluorescent signal to cross the threshold (ie, exceeds background level) (15).

Statistical analyses. Statistical tests were performed by SPSS 13.0 software (SPSS Inc., Chicago, IL, USA). Body temperature, body weight, tumor volume, and mRNA relative level of selected genes in the text and figures are mean \pm standard error of the mean (SEM). Differences between the groups in above variables across the sampling days were analyzed using a two-way analysis of variance (ANOVA) test.

The differences in the number of palpable tumors, metastases in the liver or lungs were analyzed by the $\chi^{2}$ test. The periods of temperature and mRNA relative levels of selected target genes in both groups were evaluated by the Reverse

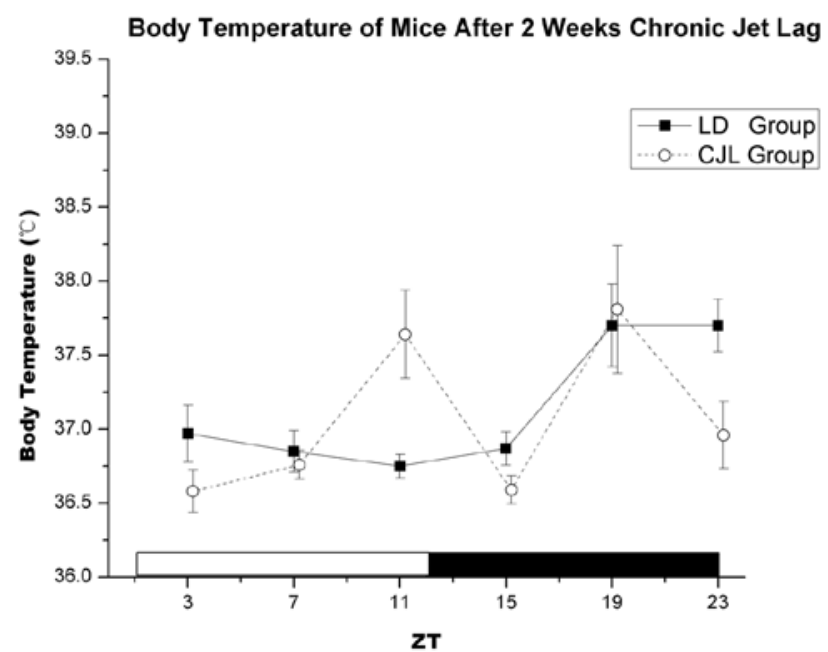

Figure 2. Circadian variation of body temperature in C57BL/6 mice after 10 days experimental chronic jet lag. Each point represents as the mean \pm SEM of 5 or 6 mice in the LD group ( $\bullet$ ) or in the CJL group (०). Open and black boxes represent light and darkness respectively for the LD mice. Body temperature in the CJL group $(n=4)$ were measured at the same time points, labeled as zeitgeber time (ZT) $3 x, 7 x, 11 x, 15 x, 19 x$, and 23x.

Elliptic Spectrum algorithm $(6,16,17)$ using the software for the Research of Biological Rhythms (Cancer Centre, Sun Yat-sen University). Body temperatures and target genes were also analyzed by cosinor analysis (18). A P-value $<0.05$ was considered to indicate statistically significant differences.

\section{Results}

Body temperature. Prior to the onset of the experimental CJL, mice in both groups displayed similar circadian rhythms according to the time and the acrophases within the darkness. Ten days after experimental CJL, the mice of the control group continued to have a clear circadian rhythm and the peak time of the body temperature was located at the darkness (period, $24 \mathrm{~h}$, cosinor analysis, $\mathrm{P}<0.0001)$. On the other hand, the mice of the CJL group changed to an ultradian rhythm with a period of $10.10 \mathrm{~h}$ (cosinor analysis, $\mathrm{P}<0.0001$; Fig. 2). No statistically significant difference of the rhythm-adjusted mean (Mesor) level of the body temperature was found between the 2 groups according to the experimental chronic jet-lag (ANOVA, $\mathrm{F}=0.093, \mathrm{P}=0.760)$.

Tumor growth. Ten days after tumor inoculation, tumors became palpable in 10 out of 24 mice in the LD group (41.6\%), and 21 out of 24 mice in the CJL group $\left(87.5 \% ; \chi^{2}=9.108, P=0.0025\right)$. Twenty-two days after tumor inoculation, prior to tissue sampling, the mean tumor volume was $777.45 \pm 115.60 \mathrm{~mm}^{3}$ in the LD group, while $1237.83 \pm 163.13 \mathrm{~mm}^{3}$ in the CJL group of mice. Prior to the animals being sacrificed, the CJL group of mice had larger tumors (two independent-samples t-test, $\mathrm{P}=0.026)$. Therefore, the tumors grew faster in the experimental CJL mice than in the LD mice (ANOVA, CJL, $\mathrm{P}=0.004$; Fig. 3).

Carcinoma metastasis. All of the samples, including the lung and tumor tissues, were examined pathologically. Metastatic 
Table I. Functional analysis of genes induced or suppressed by experimental chronic jet lag in the murine liver.

\begin{tabular}{|c|c|c|}
\hline GO ID & Gene ontology & Genes \\
\hline \multicolumn{3}{|l|}{ Induced genes } \\
\hline GO:0006637 & Acyl-CoA metabolic process & ACOT1, ACOT4, АCOT3 \\
\hline GO:0006631 & Fatty acid metabolic process & CYP4A10, CYP4A31, ACOT1, ACOT4, АCOT3 \\
\hline GO:0043066 & Negative regulation of apoptosis & VNN1, BCL6, HSPA1B, ANGPTL4 \\
\hline GO:0043069 & Negative regulation of programmed cell death & VNN1, BCL6, HSPA1B, ANGPTL4 \\
\hline GO:0060548 & Negative regulation of cell death & VNN1, BCL6, HSPA1B, ANGPTL4 \\
\hline GO:0043086 & Negative regulation of catalytic activity & GADD45G, HSPA1B, ANGPTL4 \\
\hline GO:0042981 & Regulation of apoptosis & KLF10, VNN1, BCL6, HSPA1B, ANGPTL4 \\
\hline GO:0043067 & Regulation of programmed cell death & KLF10, VNN1, BCL6, HSPA1B, ANGPTL4 \\
\hline GO:0010941 & Regulation of cell death & KLF10, VNN1, BCL6, HSPA1B, ANGPTL4 \\
\hline GO:0044092 & Negative regulation of molecular function & GADD45G, HSPA1B, ANGPTL4 \\
\hline GO:0001676 & Long-chain fatty acid metabolic process & ACOT1, ACOT3 \\
\hline GO:0006732 & Coenzyme metabolic process & ACOT1, ACOT4, ACOT3 \\
\hline GO:0055114 & Oxidation reduction & $\begin{array}{l}\text { CYP4A10, HSDL2, CYP2B9, CYP4A31, CYP4A14, } \\
\text { CYP2C } 38\end{array}$ \\
\hline GO:0006094 & Gluconeogenesis & G6PC, PCK1 \\
\hline GO:0051346 & Negative regulation of hydrolase activity & HSPA1B, ANGPTL4 \\
\hline GO:0045597 & Positive regulation of cell differentiation & KLF10, VNN1, DMBT1 \\
\hline GO:0006090 & Pyruvate metabolic process & G6PC, PCK1 \\
\hline GO:0019319 & Hexose biosynthetic process & G6PC, PCK1 \\
\hline GO:0051186 & Cofactor metabolic process & ACOT1, ACOT4, ACOT3 \\
\hline GO:0051336 & Regulation of hydrolase activity & BCL6, HSPA1B, ANGPTL4 \\
\hline GO:0046364 & Monosaccharide biosynthetic process & G6PC, PCK1 \\
\hline GO:0051094 & Positive regulation of developmental process & KLF10, VNN1, DMBT1 \\
\hline GO:0006641 & Triglyceride metabolic process & G6PC, PCK1 \\
\hline GO:0046165 & Alcohol biosynthetic process & G6PC, PCK1 \\
\hline GO:0006639 & Acylglycerol metabolic process & G6PC, PCK1 \\
\hline GO:0006662 & Glycerol ether metabolic process & G6PC, PCK1 \\
\hline GO:0006638 & Neutral lipid metabolic process & G6PC, PCK1 \\
\hline GO:0045580 & Regulation of $\mathrm{T}$ cell differentiation & VNN1, BCL6 \\
\hline GO:0018904 & Organic ether metabolic process & G6PC, PCK1 \\
\hline
\end{tabular}

Suppressed genes

GO:0000279 M phase

GO:0022403 Cell cycle phase

GO:0000280 Nuclear division

GO:0007067 Mitosis

GO:0000087 M phase of mitotic cell cycle

GO:0048285 Organelle fission

GO:0051301 Cell division

GO:0022402 Cell cycle process

GO:0000278 Mitotic cell cycle

GO:0007049 Cell cycle
CCNB1, FMN2, CCNB2, NUF2, CDC20, BIRC5, ANLN, CEP55, CDCA5, CCNA2, HELLS, RAD51 CCNB1, FMN2, CCNB2, NUF2, CDC20, BIRC5, ANLN, CEP55, CDCA5, CCNA2, HELLS, RAD51 CCNB1, CCNB2, NUF2, CDC20, BIRC5, ANLN, CEP55, CDCA5, CCNA2, HELLS

CCNB1, CCNB2, NUF2, CDC20, BIRC5, ANLN, CEP55, CDCA5, CCNA2, HELLS

CCNB1, CCNB2, NUF2, CDC20, BIRC5, ANLN, CEP55, CDCA5, CCNA2, HELLS

CCNB1, CCNB2, NUF2, CDC20, BIRC5, ANLN, CEP55, CDCA5, CCNA2, HELLS

CCNB1, FMN2, CCNB2, NUF2, CDC20, BIRC5, ANLN, CEP55, CDCA5, CCNA2, HELLS CCNB1, FMN2, CCNB2, NUF2, CDC20, BIRC5, ANLN, CEP55, CDCA5, CCNA2, HELLS, RAD51 CCNB1, CCNB2, NUF2, CDC20, BIRC5, ANLN, CEP55, CDCA5, CCNA2, HELLS

CCNB1, FMN2, CCNB2, NUF2, CDC20, BIRC5, ANLN, CEP55, CDCA5, CCNA2, HELLS, RAD51 
Table I. Continued.

\begin{tabular}{|c|c|c|}
\hline GO ID & Gene ontology & Genes \\
\hline GO:0006953 & Acute-phase response & SAA2, SAA1, ORM2, FN1 \\
\hline GO:0051303 & Establishment of chromosome localization & FMN2, BIRC5, CDCA5 \\
\hline GO:0050000 & Chromosome localization & FMN2, BIRC5, CDCA5 \\
\hline GO:0007059 & Chromosome segregation & FMN2, NUF2, BIRC5, CDCA5 \\
\hline GO:0002526 & Acute inflammatory response & SAA2, SAA1, ORM2, FN1 \\
\hline GO:0000910 & Cytokinesis & FMN2, BIRC5, ANLN \\
\hline GO:0006879 & Cellular iron ion homeostasis & HAMP2, HAMP, SCARA5 \\
\hline GO:0006952 & Defense response & $\begin{array}{l}\text { HAMP2, SAA2, HAMP, SAA1, CLEC2H, ORM2, } \\
\text { FN1 }\end{array}$ \\
\hline GO:0051656 & Establishment of organelle localization & FMN2, BIRC5, CDCA5 \\
\hline GO:0055072 & Iron ion homeostasis & HAMP2, HAMP, SCARA5 \\
\hline GO:0044270 & Nitrogen compound catabolic process & ALDH1L1, NUDT7, UPP2 \\
\hline GO:0046700 & Heterocyclic catabolic process & ALDH1L1, NUDT7, MOXD1 \\
\hline GO:0051640 & Organelle localization & FMN2, BIRC5, CDCA5 \\
\hline GO:0050832 & Defense response to fungus & HAMP2, НAMP \\
\hline GO:0006631 & Fatty acid metabolic process & SCD1, LYPLA2, ACACB, AACS \\
\hline GO:0007010 & Cytoskeleton organization & FMN2, NISCH, NUF2, BIRC5, PSTPIP2 \\
\hline GO:0055114 & Oxidation reduction & $\begin{array}{l}\text { SCD1, CYP2C55, ALDH1L1, RRM2, HSD3B5, } \\
\text { CYP2B10, MOXD1 }\end{array}$ \\
\hline GO:0007017 & Microtubule-based process & FMN2, NUF2, BIRC5, KIF20A \\
\hline GO:0009132 & Nucleoside diphosphate metabolic process & RRM2, NUDT7 \\
\hline GO:0006954 & Inflammatory response & SAA2, SAA1, ORM2, FN1 \\
\hline GO:0009620 & Response to fungus & HAMP2, НAMP \\
\hline GO:0000226 & Microtubule cytoskeleton organization & FMN2, NUF2, BIRC5 \\
\hline GO:0019058 & Viral infectious cycle & D17H6S56E-5, EG665955 \\
\hline GO:0006259 & DNA metabolic process & RRM2, SFPQ, HELLS, RAD51, NFIB \\
\hline GO:0022415 & Viral reproductive process & D17H6S56E-5, EG665955 \\
\hline GO:0030005 & Cellular di-, tri-valent inorganic cation homeostasis & HAMP2, HAMP, SCARA5 \\
\hline
\end{tabular}

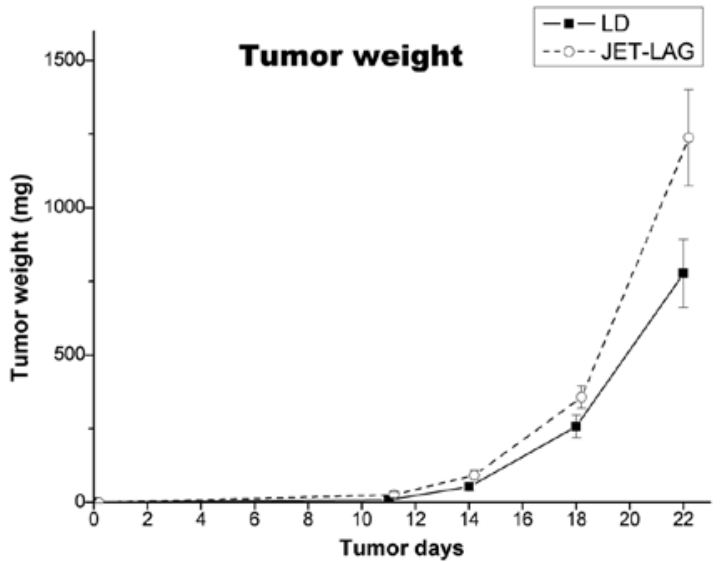

Figure 3. Tumor volume (mean $\pm \mathrm{SE}$ ) after inoculation with Lewis lung carcinoma (LLC) on Day 0. LD, control group; CJL, chronic jet lag group. Effect of the photoperiodic regimen on tumor growth, ANOVA, $\mathrm{P}=0.004$.

tumor was found on the surface of the lung in one mouse of the LD group, and in four mice of the CJL group. The lung
H\&E-stained sections showed that 10 out of 24 mice had lung metastases in the CJL group, compared to only three mice with lung metastases in the LD group $\left(\chi^{2}=5.17, \mathrm{P}=0.023\right)$.

cDNA microarray of the tumor and liver. The whole genome cDNA microarrays, which had $\sim 14,000$ well-identified genes, were used to detect the effects of the circadian disruption caused by experimental CJL on gene expression in the liver and tumor. The microarray data showed that circadian rhythm had an effect on gene expression, not only for the clock genes, but also for many other genes, such as the genes involved in angiogenesis, the cell cycle, DNA repair, and signal transduction. In the livers of the LD mice, the cDNA microarray data showed that the expression of 189 genes was higher at ZT7 (light) compared to ZT19 (darkness), while the expression of 159 genes was lower. In the tumors of the LD mice, the expression of 130 genes was higher at ZT7 (light) compared to ZT19 (darkness), while the expression of 372 genes was lower.

Gene expression in both the liver and the tumor were also altered by the experimental CJL. Cluster analysis showed that a total of 130 genes were involved in the circadian disrup- 
Table II. Functional analysis of genes induced or suppressed by experimental chronic jet lag in Lewis lung tumor.

GO ID Gene ontology Genes

Induced genes GO:0006955

Immune response

GO:0019882

GO:0002504

GO:0006952

GO:0009967

GO:0010647

GO:0045860

GO:0006954

GO:0002252

GO:0009611

GO:0033674

GO:0051347

GO:0019221

GO:0030177

GO:0043406

GO:0043085

GO:0045059

GO:0032268

GO:0045060

GO:0043368

GO:0043383

GO:0002449

GO:0044093

GO:0045859

GO:0002250

GO:0002460

GO:0043405

GO:0043549

GO:0021846

GO:0007159

GO:0050853

GO:0002443

GO:0051338

GO:0045061

GO:0043408

GO:0051251

GO:0019886

GO:0002495

GO:0002696

GO:0050867

GO:0007507

GO:0042330

GO:0006935

Defense response

Taxis

Chemotaxis
Antigen processing and presentation

Antigen processing and presentation of peptide

or polysaccharide antigen via MHC class II

Positive regulation of signal transduction

Positive regulation of cell communication

Positive regulation of protein kinase activity

Inflammatory response

Immune effector process

Response to wounding

Positive regulation of kinase activity

Positive regulation of transferase activity

Cytokine-mediated signaling pathway

Positive regulation of Wnt receptor signaling pathway

Positive regulation of MAP kinase activity

Positive regulation of catalytic activity

Positive thymic T cell selection

Regulation of cellular protein metabolic process

Negative thymic T cell selection

Positive T cell selection

Negative T cell selection

Lymphocyte mediated immunity

Positive regulation of molecular function

Regulation of protein kinase activity

Adaptive immune response

Adaptive immune response based on somatic

recombination of immune receptors built from

immunoglobulin superfamily domains

Regulation of MAP kinase activity

Regulation of kinase activity

Cell proliferation in forebrain

Leukocyte adhesion

B cell receptor signaling pathway

Leukocyte mediated immunity

Regulation of transferase activity

Thymic T cell selection

Regulation of MAPKKK cascade

Positive regulation of lymphocyte activation

Antigen processing and presentation of exogenous

peptide antigen via MHC class II

Antigen processing and presentation of peptide

antigen via $\mathrm{MHC}$ class II

Positive regulation of leukocyte activation

Positive regulation of cell activation

Heart development
H2-EA, ICAM1, PTPRC, CCR5, SERPINA3G, CCR2, H2-EB1, TLR1, CXCL9, TGTP1, IGH-6, RMCS5

H2-EA, ICAM1, H2-EB1, IGH-6, RMCS5

H2-EA, H2-EB1, RMCS5

H2-EA, PTPRC, CCR5, STAB1, CR2, TLR1, CXCL9, COTL1

HHEX, PTPRC, DOCK2, ZEB2, IGH-6

HHEX, PTPRC, DOCK2, ZEB2, IGH-6

PTPRC, TLR1, ZEB2, IGH-6

CCR5, STAB1, CCR2, TLR1, CXCL9

H2-EA, ICAM1, PTPRC, IGH-6

CCR5, STAB1, CCR2, TLR1, CXCL9, PAPSS2

PTPRC, TLR1, ZEB2, IGH-6

PTPRC, TLR1, ZEB2, IGH-6

IIGP1B, CCR2, LIFR

HHEX, ZEB2

PTPRC, ZEB2, IGH-6

PTPRC, PTGER4, TLR1, ZEB2, IGH-6

PTPRC, DOCK2

PTPRC, CPEB2, ZEB2, IGH-6,QK

PTPRC, DOCK2

PTPRC, DOCK2

PTPRC, DOCK2

H2-EA, ICAM1, IGH-6

PTPRC, PTGER4, TLR1, ZEB2, IGH-6

PTPRC, TLR1, ZEB2, IGH-6

H2-EA, ICAM1, IGH-6

H2-EA, ICAM1, IGH-6

PTPRC, ZEB2, IGH-6

PTPRC, TLR1, ZEB2, IGH-6

HHEX, ZEB2

ICAM1, PTPRC

PTPRC, IGH-6

H2-EA, ICAM1, IGH-6

PTPRC, TLR1, ZEB2, IGH-6

PTPRC, DOCK2

PTPRC, ZEB2, IGH-6

H2-EA, PTPRC, IGH-6

H2-EA, H2-EB1

H2-EA, H2-EB1

H2-EA, PTPRC, IGH-6

H2-EA, PTPRC, IGH-6

MEF2C, PTPRJ, HHEX, CHD7

C3AR1, DOCK2, EAR3

C3AR1, DOCK2, EAR3 
Table II. Continued.

\begin{tabular}{|c|c|c|}
\hline GO ID & Gene ontology & Genes \\
\hline GO:0045058 & $\mathrm{T}$ cell selection & PTPRC, DOCK2 \\
\hline GO:0007243 & Protein kinase cascade & PTPRC, TLR1, MAPK8, IGH-6 \\
\hline GO:0000165 & MAPKKK cascade & PTPRC, MAPK8, IGH-6 \\
\hline GO:0007626 & Locomotory behavior & C3AR1, DOCK2, EAR3, CHD7 \\
\hline GO:0016525 & Negative regulation of angiogenesis & HHEX, STAB 1 \\
\hline GO:0030890 & Positive regulation of $\mathrm{B}$ cell proliferation & PTPRC, IGH-6 \\
\hline GO:0002478 & $\begin{array}{l}\text { Antigen processing and presentation of exogenous } \\
\text { peptide antigen }\end{array}$ & H2-EA, H2-EB1 \\
\hline GO:0019835 & Cytolysis & GZME, FGL2 \\
\hline \multicolumn{3}{|c|}{ Suppressed genes } \\
\hline GO:0030005 & Cellular di-, tri-valent inorganic cation homeostasis & ALAS2, HIF1A, MT2, MT1, APLP2 \\
\hline GO:0006873 & Cellular ion homeostasis & ALAS2, HIF1A, SNCA, MT2, MT1, APLP2 \\
\hline GO:0055066 & Di-, tri-valent inorganic cation homeostasis & ALAS2, HIF1A, MT2, MT1, APLP2 \\
\hline GO:0042592 & Homeostatic process & $\begin{array}{l}\text { HBA-A1, HSPA1L, ALAS2, HIF1A, SNCA, MT2, } \\
\text { MT1, APLP2 }\end{array}$ \\
\hline GO:0055082 & Cellular chemical homeostasis & ALAS2, HIF1A, SNCA, MT2, MT1, APLP2 \\
\hline GO:0030003 & Cellular cation homeostasis & ALAS2, HIF1A, MT2, MT1, APLP2 \\
\hline GO:0050801 & Ion homeostasis & ALAS2, HIF1A, SNCA, MT2, MT1, APLP2 \\
\hline GO:0055080 & Cation homeostasis & ALAS2, HIF1A, MT2, MT1, APLP2 \\
\hline GO:0019725 & Cellular homeostasis & ALAS2, HIF1A, SNCA, MT2, MT1, APLP2 \\
\hline GO:0019835 & Cytolysis & GZMD, GZMF, GZMG \\
\hline GO:0048878 & Chemical homeostasis & ALAS2, HIF1A, SNCA, MT2, MT1, APLP2 \\
\hline GO:0007626 & Locomotory behavior & CCL3, S100A8, SNCA, S100A9, APLP2 \\
\hline GO:0007263 & Nitric oxide mediated signal transduction & MT2, MT1 \\
\hline GO:0002246 & Healing during inflammatory response & HIF1A, CD44 \\
\hline GO:0010273 & Detoxification of copper ion & MT2, MT1 \\
\hline GO:0035239 & Tube morphogenesis & HIF1A, ADM, CD44, GJA1 \\
\hline GO:0042541 & Hemoglobin biosynthetic process & ALAS2, HIF1A \\
\hline GO:0007610 & Behavior & CCL3, S100A8, SNCA, S100A9, APLP2 \\
\hline GO:0006882 & Cellular zinc ion homeostasis & MT2, MT1 \\
\hline GO:0046688 & Response to copper ion & MT2, MT1 \\
\hline GO:0055069 & Zinc ion homeostasis & MT2, MT1 \\
\hline GO:0030097 & Hemopoiesis & HBA-A1, ALAS2, HIF1A, HBB-B1 \\
\hline GO:0020027 & Hemoglobin metabolic process & ALAS2, HIF1A \\
\hline GO:0006091 & Generation of precursor metabolites and energy & CYBB, SNCA, PFKP, ERO1L \\
\hline GO:0035295 & Tube development & HIF1A, ADM, CD44, GJA1 \\
\hline GO:0015671 & Oxygen transport & HBA-A1, HBB-B1 \\
\hline GO:0001701 & In utero embryonic development & HBA-A1, HIF1A, ADM, GJA1 \\
\hline GO:0006935 & Chemotaxis & CCL3, S100A8, S100A9 \\
\hline GO:0042330 & Taxis & CCL3, S100A8, S100A9 \\
\hline GO:0022900 & Electron transport chain & CYBB, SNCA, ERO1L \\
\hline GO:0042060 & Wound healing & HIF1A, CD44, GJA1 \\
\hline GO:0048534 & Hemopoietic or lymphoid organ development & HBA-A1, ALAS2, HIF1A, HBB-B1 \\
\hline GO:0015669 & Gas transport & HBA-A1, HBB-B1 \\
\hline GO:0008219 & Cell death & GZMD, PDCD6IP, NIACR1, GZMF, GZMG \\
\hline GO:0002520 & Immune system development & HBA-A1, ALAS2, HIF1A, HBB-B1 \\
\hline GO:0016265 & Death & GZMD, PDCD6IP, NIACR1, GZMF, GZMG \\
\hline GO:0042981 & Regulation of apoptosis & HSPA1L, SERINC3, HIF1A, SNCA, NIACR1 \\
\hline GO:0043067 & Regulation of programmed cell death & HSPA1L, SERINC3, HIF1A, SNCA, NIACR1 \\
\hline GO:0010941 & Regulation of cell death & HSPA1L, SERINC3, HIF1A, SNCA, NIACR1 \\
\hline GO:0009611 & Response to wounding & CCL3, HIF1A, CD44, GJA1 \\
\hline
\end{tabular}


Table II. Continued.

\begin{tabular}{lll}
\hline GO ID & \multicolumn{1}{c}{ Gene ontology } & \multicolumn{1}{c}{ Genes } \\
\hline GO:0034599 & Cellular response to oxidative stress & HIF1A, SNCA \\
GO:0001947 & Heart looping & HIF1A, GJA1 \\
GO:0009636 & Response to toxin & MT2, MT1 \\
GO:0006879 & Cellular iron ion homeostasis & ALAS2, HIF1A \\
GO:0033554 & Cellular response to stress & HSPA1L, HIF1A, SNCA, ERO1L \\
GO:0043009 & Chordate embryonic development & HBA-A1, HIF1A, ADM, GJA1 \\
GO:0002274 & Myeloid leukocyte activation & SNCA, NDRG1 \\
GO:0055072 & Iron ion homeostasis & ALAS2, HIF1A \\
GO:0009792 & Embryonic development ending in birth or egg hatching & HBA-A1, HIF1A, ADM, GJA1 \\
GO:0044057 & Regulation of system process & SNCA, GJA1, NIACR1
\end{tabular}

$\mathbf{A}$

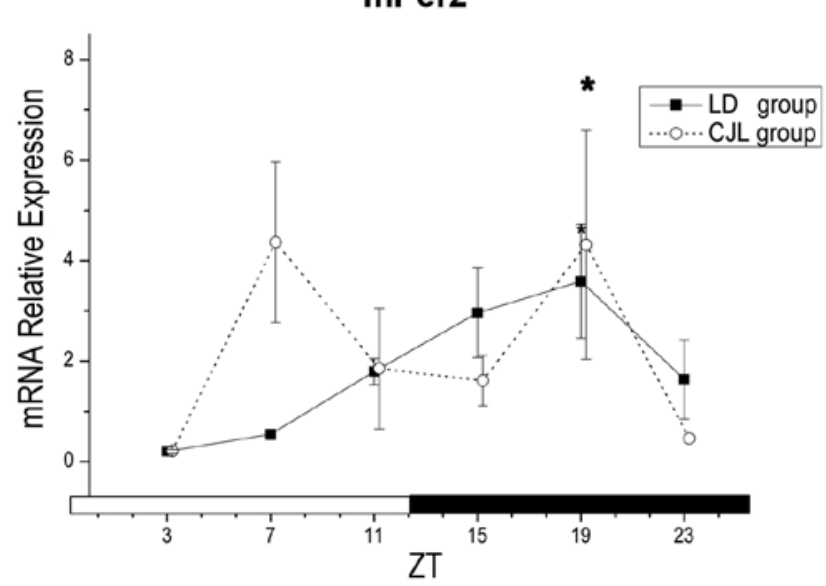

B

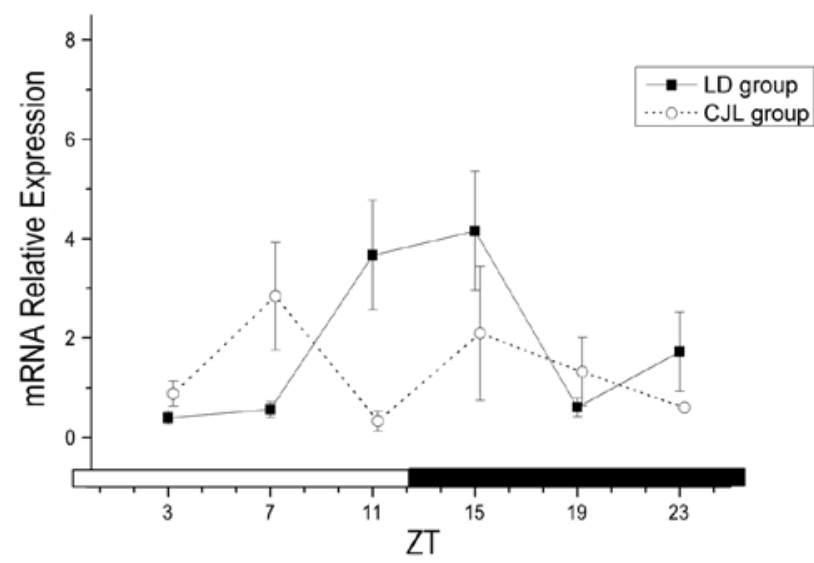

Figure 4. Circadian variation of mPer2 expression in the liver and tumors of mice. (A) Circadian variation of mPer2 expression in liver. (B) Circadian variation of mPer2 expression in tumor. ${ }^{*} \mathrm{P}<0.05$ significantly different from ZT3 as per one-way ANOVA with least significant difference test (LSD) in the LD group mice.

tion in the liver (43 genes induced and 87 genes suppressed), and 142 genes were involved in that of the tumor (100 genes induced and 42 genes suppressed; Tables I and II).

Effects of the experimental chronic jet lag on selected genes expression. The selected gene expression profiles of mPer2, PTPRC, CD44, NDRG1 were detected using the real-time quantitative RT-PCR method.

The effects of experimental CJL on mRNA expression of clock gene mPer2 in the mice livers are shown in Fig. 4A and Table III. The animals showed a clear circadian rhythm in the LD group of mice (cosinor analysis, $\mathrm{P}=0.0009$ ) and the peak time was located at ZT16:57 (14:34-19:28). In the CJL group of mice, CJL markedly altered the circadian expression patterns of mPer2 (Fig. 4A) from a circadian rhythm to a ultradian rhythm for CJL mice (period, $10.10 \mathrm{~h}$, cosinor analysis, $\mathrm{P}=0.058$ ). Nevertheless, CJL also clearly altered the $24-h$ patterns of mPer2 expressions in the tumor tissue. In the tumors of the LD group of mice, the 24-h rhythmic pattern in mPer2 were still observed, and the peak was located at the ZT13:00 (09:20-16:42) (cosinor analysis, $\mathrm{P}=0.0224$ ) (Fig. 4B). However, CJL ablated the rhythmic changes in mPer2 expression in the tumor (cosinor analysis, $\mathrm{P}>0.05$ ) (Fig. 4B, Table III).
The selected gene expression profiles of PTPRC, CD44, and NDRG1 in the tumor, which were identified by the microarray, were also detected using the real-time quantitative RT-PCR method. Neither the rhythm nor the effect of the CJL was found in mRNA expression of PTPRC and CD44 (Fig. 5, Table IV). However, the mRNA expression level of NDRG1 in the tumor was suppressed by the experimental CJL even though the result was not statistically significant $(\mathrm{P}=0.093)$. The Mesor level of NDRG1 in the LD group of mice was 3.501 \pm 3.677 and it was $0.998 \pm 0.503$ in the CJL group of mice. No circadian rhythm of the NDRG1 expression was found in either group of mice (cosinor analysis, $\mathrm{P}>0.05$ ) (Fig. 5, Table IV).

\section{Discussion}

The correlation between the disruption of circadian rhythm and the development of cancer has been identified in both rodentmodel and human research studies. These have suggested that circadian rhythm may be an important control point in tumorigenesis and growth. In this study, experimental CJL, produced by an 8 -h shift of the light-dark cycle every 2 days, was used to maximally disturb the circadian rhythm of the experimental group. The body temperature of the mice and the clock gene, 
Table III. Cosinor analyses of mPer2 genes expression in liver and tumor of C57BL/6 male mice.

\begin{tabular}{cccccr}
\hline & & & \multicolumn{2}{c}{ Cosinor analysis } \\
\cline { 5 - 6 } Tissue & Mesor \pm SEM & Period (h) & Acrophase (95\% CI) (h:min) & Amplitude (95\% CI) & P-value \\
\hline Liver & & & & & \\
LD & $1.801 \pm 0.543$ & 24 & $16: 57(14: 34-19: 28)$ & $1.658(0.719-2.598)$ & 0.0009 \\
CJL & $2.207 \pm 1.197$ & 10.10 & $07: 45$ & 1.963 & 0.0582 \\
Tumor & & & & & $1.768(0.260-3.277)$ \\
LD & $1.543 \pm 0.784$ & 24 & $13: 00(09: 20-16: 42)$ & 1.369 & 0.0224 \\
CJL & $1.502 \pm 0.981$ & 10.30 & $06: 27$ & & 0.1383 \\
\hline
\end{tabular}

LD, control $12 \mathrm{~h}$ light $12 \mathrm{~h}$ dark group; CJL, chronic jet lag group

Table IV. Cosinor analyses of selected genes (PTPRC, CD44 and NDRG1) expression in the tumor of C57BL/6 male mice.

\begin{tabular}{lllllr}
\hline & & & \multicolumn{2}{c}{ Cosinor analysis } \\
\cline { 5 - 6 } Variable & Mesor \pm SEM & Period $(\mathrm{h})$ & Acrophase $(95 \% \mathrm{CI})(\mathrm{h}: \mathrm{min})$ & Amplitude (95\% CI) & P-value \\
\hline PTPRC & & & & & \\
LD & $1.612 \pm 0.463$ & 12 & $00: 28(03: 24-09: 59)$ & $0.806(0.011-1.643)$ & 0.0468 \\
CJL & $1.272 \pm 0.428$ & 21.7 & $00: 00$ & 0.521 & 0.2568 \\
CD44 & & & & & 0.197 \\
LD & $1.488 \pm 0.754$ & 24 & $13: 46$ & 0.439 & 0.9320 \\
CJL & $1.331 \pm 0.388$ & 23 & $01: 10$ & & 0.3705 \\
NDRG1 & & & $10: 41$ & 5.920 & 0.1032 \\
LD & $3.501 \pm 3.667$ & 24 & $04: 21$ & 0.343 & 0.6253 \\
CJL & $0.998 \pm 0.503$ & 12.80 & &
\end{tabular}

LD, control $12 \mathrm{~h}$ light $12 \mathrm{~h}$ dark group; CJL, chronic jet lag group

mPer2, were used to monitor the effect of the experimental CJL on their circadian rhythms. After 10 days of experimental CJL, the rhythmic profile of the body temperature of the mice was changed from a circadian rhythm to an ultradian rhythm, and the period was shortened from $24 \mathrm{~h}$ to $10.10 \mathrm{~h}$. At the mRNA level, CJL also markedly altered the circadian expression patterns of mPer2 in the livers from a circadian rhythm to a suggestive ultradian rhythm. Our results are supported by previous reports which have shown that CJL can disrupt the circadian rhythm, including body temperature, local activity, hematological parameters, and even gene expression and the immune system $(6,9)$.

Circadian disruption also accelerated the growth of the inoculated LLC in our C57BL/6 mice. This result is consistent with previous reports as circadian disruption has shown the same effect on Glasgow osteosarcoma (GOS) or pancreatic adenocarcinoma (PO3) $(9,10)$. The rhythm disorder also increased the rate of cancer metastasis to the lungs, which was $41.67 \%$ in mice of the CJL group, but only $12.5 \%$ in the LD group $(\mathrm{P}<0.05)$.

In order to investigate the possible molecular reasons underlying the acceleration of tumor growth and induction of metastases caused by circadian disruption, the whole genome scan method was used to detect the effect of circadian rhythm and rhythm disruption on gene expression within the liver and tumor. In the liver, a total of 348 genes were clock-controlled genes (gene expression at ZT19 compared to ZT7 was increased in 159 genes and decreased in 189). The total number of clockcontrolled genes was consistent with the studies of Duffield (19), Panda et al (20) (335 genes) and Ueda et al (21) (393 genes). In the tumor of the LD group of mice, 372 genes were induced at ZT19 compared to the ZT7, while 130 genes were suppressed; therefore, more genes in the tumor (502 genes in total) were clock-controlled genes.

Experimental CJL also altered the gene expression in both the liver and the tumor. The cluster analysis showed that in the liver a total number of 659 genes were involved in the circadian disruption, and in the tumor, 609 genes. Among the genes involved in the liver, 37 were cell cycle genes as categorized by the gene ontology (GO) biological function, 2 genes (Per2 and Dbp) were circadian exercise genes, and 15 genes were immune system process genes (Fig. 6). Among those involved in the tumor, 4 genes were cell cycle genes, 1 gene (epidermal growth factor receptor, EGFR) was a circadian exercise gene, and 27 genes were immune system process genes (Fig. 7). 
A

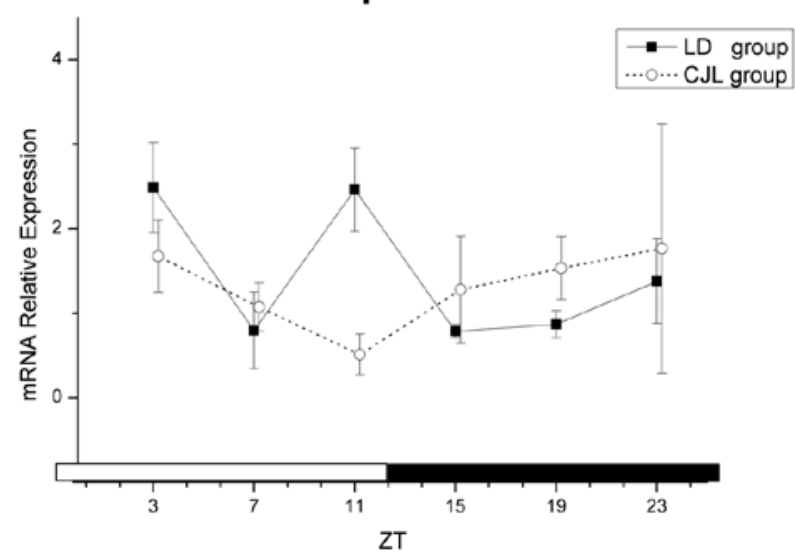

B

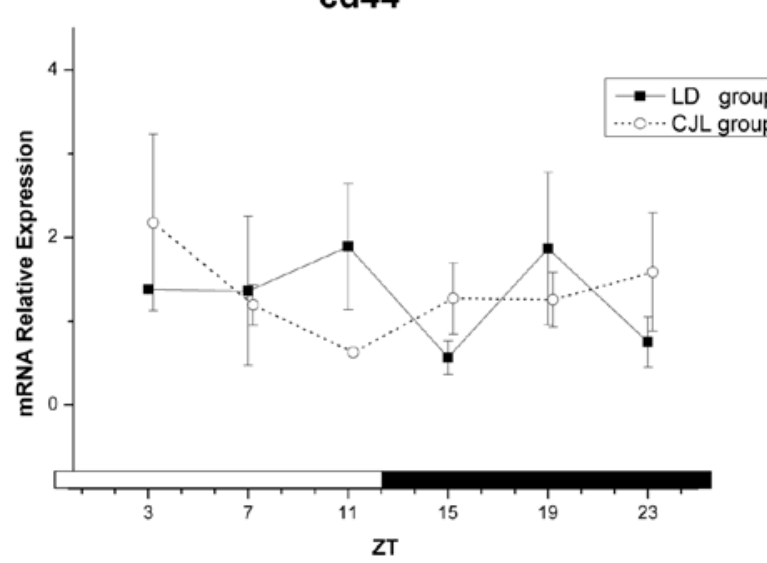

C

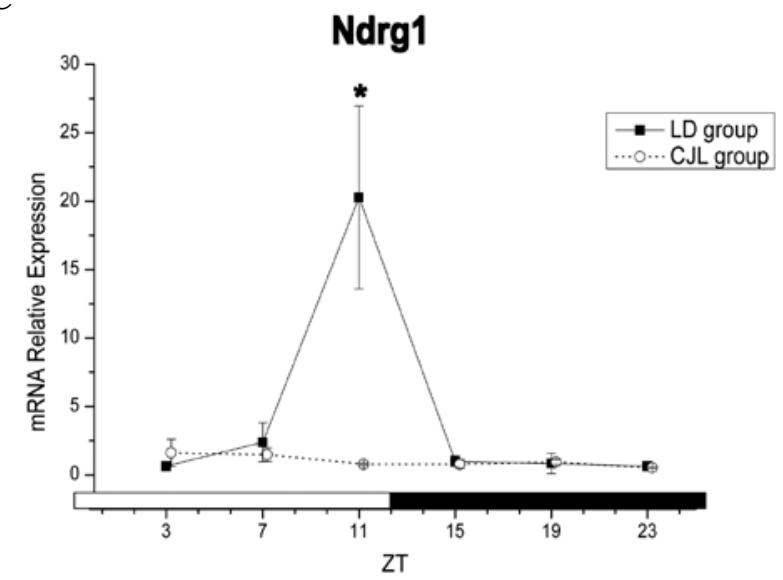

Figure 5. Circadian variation of genes expression in tumors of mice. (A) PTPRC; (B) CD44; (C) NDRG1. "P<0.05 significantly different from ZT3 as per one-way ANOVA with least significant difference test (LSD) in the LD group mice.

In the liver, genes related to the peroxisome proliferatoractivated receptors (PPAR) signaling pathway; the cell cycle and the p53 signaling pathway, including CCNA1, CCNB1, CCNB2, and CYP4A were involved. In the tumor, genes related to the following areas were identified, including the cell cycle (GZMD, PDCD6IP, NIACR1, GZMF and GZMG), apoptosis (HSPA1L, SERINC3, HIF1A, SNCA and NIACR1),
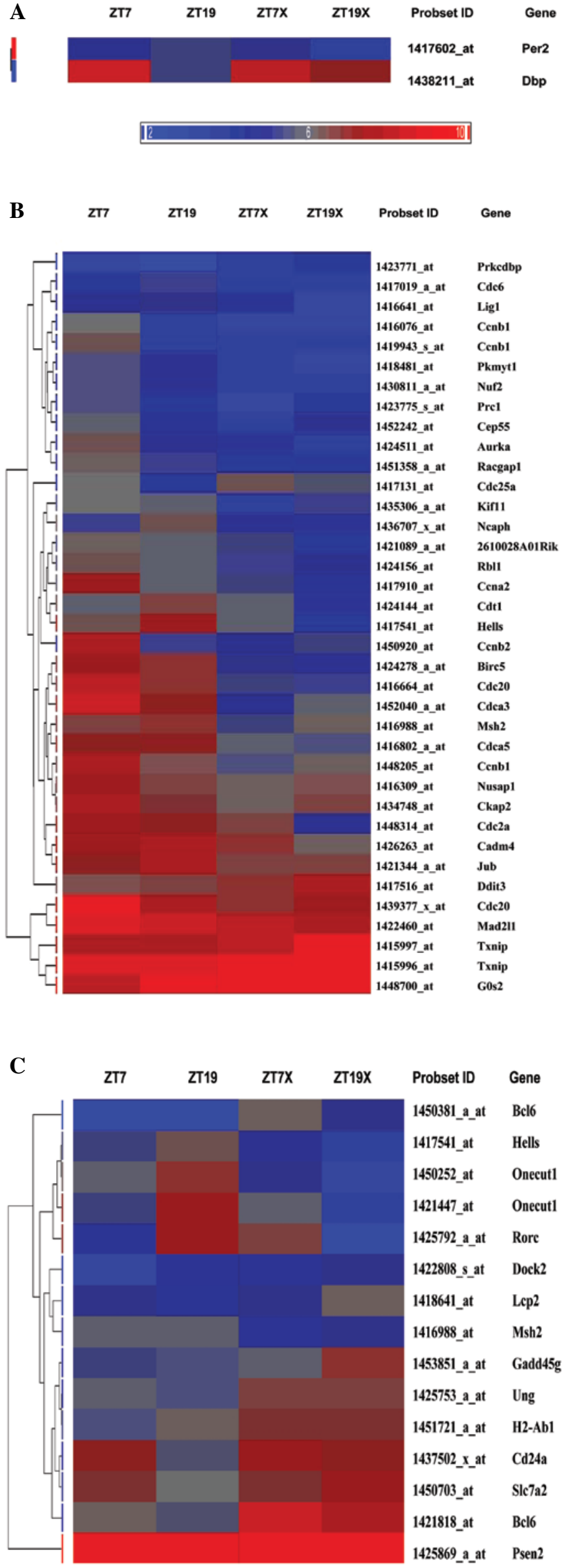

Figure 6. Cluster analysis of gene ontology (GO) biological functions identified genes involved in the circadian disruption in the liver. (A) Cell cycle genes, (B) circadian exercise genes, (C) immune system process genes. 
A
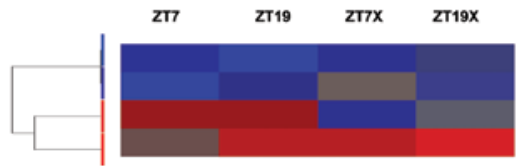

Probset

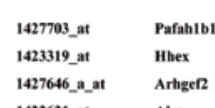

B

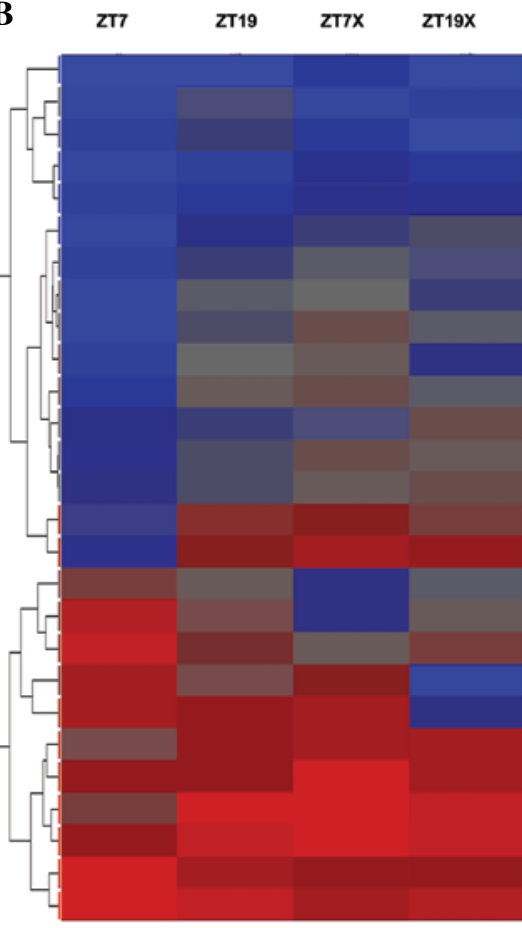

Figure 7. Cluster analysis of gene ontology (GO) biological functions identified genes involved in the circadian disruption in tumors. (A) Cell cycle genes, (B) immune system process genes.

the immune response (H2-EA, ICAM1, PTPRC, CCR5, SERPINA3G, CCR2, H2-EB1, TLR1, CXCL9, TGTP1, IGH-6 and RMCS5), the Wnt signaling pathway (HHEX and ZEB2) and the MAPK signaling pathway (PTPRC, ZEB2, IGH-6 and MAPK8). These genes (shown in Tables I and II) have close relationships with tumor growth, so they may constitute the molecular mechanisms underlying the promotion of tumor growth by disruption of circadian rhythm.

Tumor metastasis is an important clinical problem contributing to the majority of cancer-related deaths. One metastasis suppressor gene, N-myc downstream regulated 1 (NDRG1) was found to be supressed in the CJL mice. The Mesor level of the NDRG1 mRNA expression in the tumor was suppressed by the experimental CJL by about 3 -fold. NDRG1 has been shown to be involved in p53-mediated apoptosis and to be regulated by the phosphatase and tensin homolog (PTEN) gene. Its expression was shown to be negatively correlated with tumor metastasis. Studies in vitro and in vivo have demonstrated a significant reduction in the metastatic ability of cells that overexpression NDRG1 (22). This gene also affects cell cycle, apoptosis, and tumorigenesis through interactions with other proteins; the network of NDRG1 in the tumor is shown in Fig. 8. In this study, the expression of the metastasis suppressor gene, NDRG1, in the tumor was decreased by CJL. This may be one potential explanation for the induction of metastasis by circadian disruption.

In conclusion, jet lag has been shown to change the rhythmic profiles of body temperature, and to have an effect on tumor growth and metastasis. This may be due to the effect of circadian disruption on gene expression, not only of clock genes, but also of tumor-related genes, such as those involved in

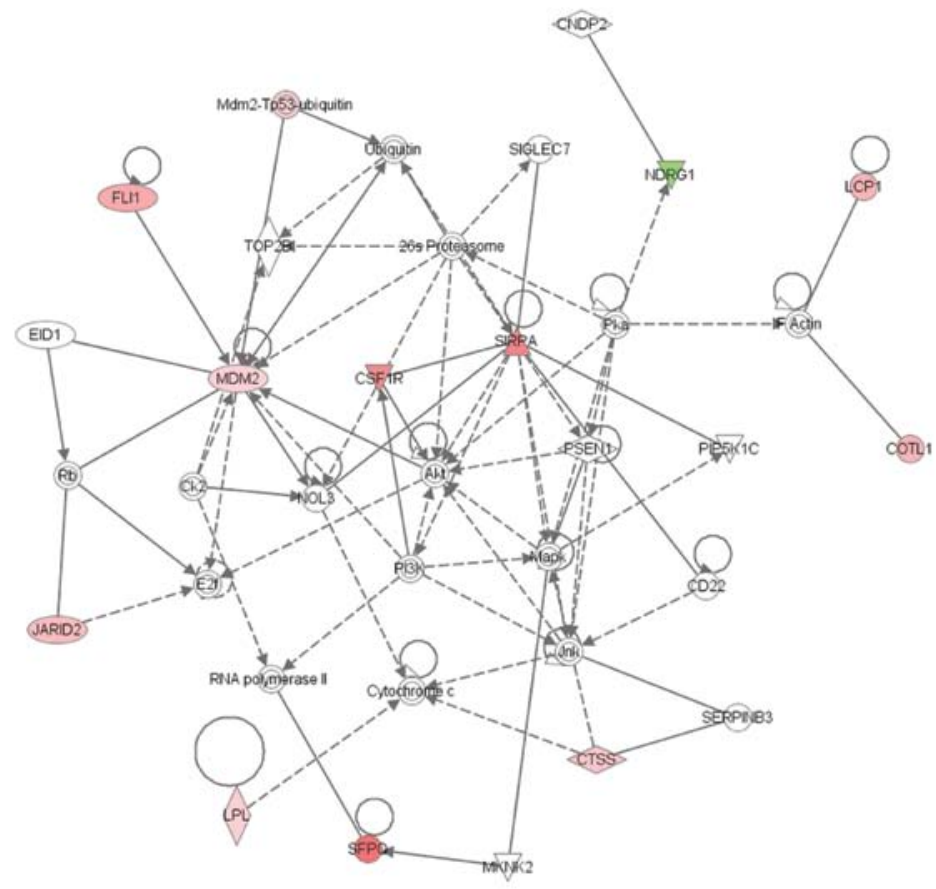

Figure 8. Network of N-myc downstream regulated 1 (NDRG1) constructed by the Ingenuity pathway analysis (IPA). Genes, proteins, and chemicals are displayed as various shapes. The shapes are indicative of the molecular class (protein family, or chemical). Coloring is based on the expression values in the CJL mice compared to the LD mice. Red indicates upregulation (positive values), green downregulation (negative values), gray that the molecule was part of the dataset but did not meet the user-specified cut-off value, and white that the molecule was added from the Ingenuity Knowledge Base. Lines connecting molecules indicate molecular relationships. Dashed lines indicate indirect interactions; solid lines indicate direct interactions. The style of the arrows indicates specific molecular relationships and the directionality of the interaction. 
the cell cycle, immunity, and cancer metastasis. It is suggested that circadian disruption can promote tumor progression and metastasis by affecting the expression of both tumor-related and metastasis suppressor genes.

\section{Acknowledgements}

We thank Yueli Sun, Bing Yang and Yanru Feng (Sun Yat-sen University) for assistance with the animal experiments; Mr. Yongju Liang (Sun Yat-sen University) for assistance with the cell culture; Mr. Shengzhao Yang (Sun Yat-sen University) for assistance with the software program of the Research of Biological Rhythms, and Dr Gregory Ford (Morehouse School of Medicine, USA) for help with microarray data analysis. We especially thank Dr Jennifer Evans (Morehouse School of Medicine, USA) for helpful discussions and writing modification. We also thank Dr X.M. Li and Dr F. Lévi (INSERM U776, France) for helpful discussions. This study was supported by the National Natural Science Foundation of China (no. 30500589, to M.W.), and by the Scientific Research Foundation for the Returned Overseas Chinese Scholars, State Education Ministry, P.R. China (2005, to M.W.).

\section{References}

1. Schibler U and Sassone-Corsi P: A web of circadian pacemakers. Cell 111: 919-922, 2002.

2. Van GR: How the clock sees the light. Nat Neurosci 11: 628-630, 2008.

3. Wu MW, Li XM, Xian LJ and Levi F: Effects of meal timing on tumor progression in mice. Life Sci 75: 1181-1193, 2004.

4. Wu MW, Xian LJ, Li XM, Pasquale I and Francis L: Circadian expression of dihydropyrimidine dehydrogenase, thymidylate synthase, c-myc and p53 mRNA in mouse liver tissue. Ai Zheng 23: 235-242, 2004.

5. Wu MW, Zeng ZL, Li S, et al: Circadian variation of plasma cortisol and whole blood reduced glutathione levels in nasopharyngeal carcinoma patients. Ai Zheng 27: 237-242, 2008.

6. Wu M, Cai Y, Sun J, et al: Effects of experimental chronic jet lag on hematological and immune parameters in mice. Biol Rhythm Res 41: 363-378, 2010.
7. Fu L and Lee CC: The circadian clock: pacemaker and tumour suppressor. Nat Rev Cancer 3: 350-361, 2003.

8. Fu L, Pelicano H, Liu J, Huang P and Lee C: The circadian gene Period2 plays an important role in tumor suppression and DNA damage response in vivo. Cell 111: 41-50, 2002.

9. Filipski E, Innominato PF, Wu M, et al: Effects of light and food schedules on liver and tumor molecular clocks in mice. J Natl Cancer Inst 97: 507-517, 2005.

10. Filipski E, King VM, Li X, et al: Host circadian clock as a control point in tumor progression. J Natl Cancer Inst 94: 690-697, 2002.

11. Wood PA, Yang X, Taber A, et al: Period 2 mutation accelerates ApcMin/+ tumorigenesis. Mol Cancer Res 6: 1786-1793, 2008.

12. Gery S, Komatsu N, Baldjyan L, Yu A, Koo D and Koeffler HP: The circadian gene perl plays an important role in cell growth and DNA damage control in human cancer cells. Mol Cell 22: 375-382, 2006.

13. Huang da W, Sherman BT and Lempicki RA: Systematic and integrative analysis of large gene lists using DAVID bioinformatics resources. Nat Protoc 4: 44-57, 2009.

14. Dennis G Jr, Sherman BT, Hosack DA, et al: DAVID: database for annotation, visualization, and integrated discovery. Genome Biol 4: P3, 2003.

15. Livak KJ and Schmittgen TD: Analysis of relative gene expression data using real-time quantitative PCR and the 2(-Delta Delta C(T)) Method. Methods 25: 402-408, 2001.

16. Mauvieux B, Gouthiere L, Sesboue B and Davenne D: A study comparing circadian rhythm and sleep quality of athletes and sedentary subjects engaged in night work. Can J Appl Physiol 28: 831-887, 2003 (In French).

17. Mauvieux B, Gouthiere L, Sesboue B, Denise P and Davenne D: Effects of the physical exercise and sports on the circadian rhythm of temperature and waking/sleep pattern of the elderly person. Examples in retired and night workers. Pathol Biol (Paris) 55: 205-207, 2007 (In French).

18. Nelson W, Tong YL, Lee JK and Halberg F: Methods for cosinorrhythmometry. Chronobiologia 6: 305-323, 1979.

19. Duffield GE: DNA microarray analyses of circadian timing: the genomic basis of biological time. J Neuroendocrinol 15: 991-1002, 2003.

20. Panda S, Antoch MP, Miller BH, et al: Coordinated transcription of key pathways in the mouse by the circadian clock. Cell 109: 307-320, 2002.

21. Ueda HR, Chen W, Adachi A, et al: A transcription factor response element for gene expression during circadian night. Nature 418: 534-539, 2002.

22. Ando T, Ishiguro H, Kimura M, et al: Decreased expression of NDRG1 is correlated with tumor progression and poor prognosis in patients with esophageal squamous cell carcinoma. Dis Esophagus 19: 454-458, 2006. 